\title{
Notes on cohomologies of ternary algebras of associative type
}

\author{
H. ATAGUEMA and A. MAKHLOUF
}

Laboratoire de Mathématiques, Informatique et Applications, Université de Haute Alsace, 4 rue des Frères Lumière F-68093 Mulhouse, France

E-mails: hammimi.ataguema@uha.fr,abdenacer.makhlouf@uha.fr

\begin{abstract}
The aim of this paper is to investigate the cohomologies for ternary algebras of associative type. We study in particular the cases of partially associative ternary algebras and weak totally associative ternary algebras. Also, we consider the Takhtajan's construction, which was used to construct a cohomology of ternary Nambu-Lie algebras using Chevalley-Eilenberg cohomology of Lie algebras, and discuss it in the case of ternary algebras of associative type. One of the main results of this paper states that a usual deformation cohomology does not exist for partially associative ternary algebras which implies that their operad is not a Koszul operad.
\end{abstract}

2000 MSC: 17A40, 55N99.

\section{Introduction}

The paper is dedicated to studying cohomologies adapted to deformation theory of ternary algebraic structures appearing more or less naturally in various domains of theoretical and mathematical physics and data processing. Indeed, theoretical physics progress of quantum mechanics and the discovery in 1973 of the Nambu mechanics (see [48]), as well as a work of Okubo on Yang-Baxter equation (see [49]), gave impulse to a significant development on ternary algebras and more generally $n$-ary algebras. The ternary operations, in particular cubic matrices, were already introduced in the nineteenth century by Cayley. The cubic matrices were considered again and generalized by Kapranov, Gelfand, and Zelevinskii in 1994 (see [30]) and Sokolov in 1972 (see [54]). Another recent motivation to study ternary operation comes from string theory and M-branes where appeared naturally a so-called Bagger-Lambert algebra (see [3]). For other physical applications, see [1, 31, 32, 33, 34, 35].

The concept of ternary algebras was introduced first by Jacobson (see [29]). In connection with problems from Jordan theory and quantum mechanics, he defined the Lie triple systems. A Lie triple system consists of a space of linear operators on vector space $V$ that is closed under the ternary bracket $[x, y, z]_{T}=[[x, y], z]$, where $[x, y]=x y-y x$. Equivalently, a Lie triple system may be viewed as a subspace of the Lie algebra closed relative to the ternary product. A Lie triple system arose also in the study of symmetric spaces (see [43]). We distinguish two kinds of generalization of binary Lie algebras: ternary Lie algebras (resp., $n$ ary Lie algebras) in which the Jacobi identity is generalized by considering a cyclic summation over $\mathcal{S}_{5}$ (resp., $\mathcal{S}_{2 n-1}$ ) instead of $\mathcal{S}_{3}$ (see $[25,47]$ ), and ternary Nambu algebras (resp., $n$-ary Nambu algebras) in which the fundamental identity generalizes the fact that the adjoint maps are derivations. The fundamental identity appeared first in Nambu mechanics (see [48]), see also [55] for the algebraic formulation of the Nambu mechanics. The abstract definitions of ternary and more generally $n$-ary Nambu algebras or $n$-ary Nambu-Lie algebras (when 
the bracket is skew-symmetric) were given by Fillipov in 1985 (see [13] in Russian). While the $n$-ary Leibniz algebras were introduced and studied in [8]. For deformation theory and cohomologies of ternary algebras of Lie type, we refer to $[14,15,26,38,56,57]$.

In another hand, ternary algebras or more generally $n$-ary algebras of associative type were studied by Carlsson, Lister, and Loos (see [6, 40,42]). The typical and founding example of totally associative ternary algebra was introduced by Hestenes (see [27]) defined on the linear space of rectangular matrices $A, B, C \in \mathcal{M}_{m, n}$ with complex entries by $A B^{*} C$ where $B^{*}$ is the conjugate transpose matrix of $B$. This operation is strictly speaking not a ternary algebra product on $\mathcal{M}_{m, n}$ as it is linear on the first and the third arguments but conjugate-linear on the second argument. The ternary operation of associative type leads to two principal classes: totally associative ternary algebras and partially associative ternary algebras. Also they admit some variants. The totally associative ternary algebras are also called associative triple systems. The operads of $n$-ary algebras were studied by Gnedbaye (see [20, 21]), see also $[22,28]$. The cohomology of totally associative ternary algebras was studied by Carlsson through the embedding (see [7]). In [2], we extended to ternary algebras of associative type, the 1-parameter formal deformations introduced by Gerstenhaber [16, 17, 18], see [44] for a review. We built a 1-cohomology and 2-cohomology of partially associative ternary algebras fitting with the deformation theory.

The generalized Poisson structures and $n$-ary Poisson brackets were discussed in $[9,10$, $24,47]$. While the quantization problem was considered in $[11,12]$. Further generalizations and related works could be found in $[4,5,23,50,51,52]$.

In this paper, we summarize in Section 2 the definitions of ternary algebras of associative type and Lie type with examples, and recall the basic settings of homological algebra. Section 3 is devoted to study the cohomology of partially associative ternary algebras with values in the algebra. We provide the first and the second coboundary operators and show that their extension to a usual 3-coboundary does not exist. This shows that the operad of partially ternary algebras is not a Koszul operad. In Section 4, we consider weak totally associative ternary algebras for which we construct a $p$-coboundary operator extending, to any $p$, the 2-coboundary operators already defined by Takhtajan (see [56]). In Section 5, we discuss Takhtajan's construction for ternary algebras of associative type. The process was introduced by Takhtajan to construct a cohomology for ternary algebras of Lie type starting from a cohomology of binary algebras. It was used to derive the cohomology of ternary Nambu-Lie algebras from the Chevalley-Eilenberg cohomology of Lie algebras. We show that a usual cohomology of ternary algebras of partially associative type cannot be constructed from binary algebras of associative type. We also show in Section 6, that the skew-associative binary algebras do not carry a usual cohomology fitting with deformation theory and therefore their operad is not Koszul as well.

\section{Generalities}

In this section, we summarize the definitions of different ternary algebra structures of associative type and Lie type and provide some examples, and then give general settings for cohomology theories.

\subsection{Ternary algebra structures}

Let $\mathbb{K}$ be an algebraically closed field of characteristic zero and let $V$ be a $\mathbb{K}$-vector space. A ternary operation on $V$ is a linear map $m: V \otimes V \otimes V \longrightarrow V$ or a trilinear map $m$ : $V \times V \times V \longrightarrow V$. If $V$ is $n$-dimensional vector space and $B=\left\{e_{1}, \ldots, e_{n}\right\}$ is a basis of $V$, 
the ternary operation $m$ is completely determined by its structure constants $\left\{C_{i j k}^{s}\right\}$, where

$$
m\left(e_{i} \otimes e_{j} \otimes e_{k}\right)=\sum_{s=1}^{n} C_{i j k}^{s} e_{s}
$$

A ternary operation is said to be symmetric (resp., skew-symmetric) if

$$
m\left(x_{\sigma(1)} \otimes x_{\sigma(2)} \otimes x_{\sigma(3)}\right)=m\left(x_{1} \otimes x_{2} \otimes x_{3}\right), \quad \forall \sigma \in \mathcal{S}_{3} \text { and } \forall x_{1}, x_{2}, x_{3} \in V
$$

and, respectively,

$$
m\left(x_{\sigma(1)} \otimes x_{\sigma(2)} \otimes x_{\sigma(3)}\right)=\operatorname{Sgn}(\sigma) m\left(x_{1} \otimes x_{2} \otimes x_{3}\right), \quad \forall \sigma \in \mathcal{S}_{3} \text { and } \forall x_{1}, x_{2}, x_{3} \in V
$$

where $\operatorname{Sgn}(\sigma)$ denotes the signature of the permutation $\sigma \in \mathcal{S}_{3}$.

We have the following type of "associative" ternary operations.

Definition 2.1. A totally associative ternary algebra is given by a $\mathbb{K}$-vector space $V$ and a ternary operation $m$ satisfying, for every $x_{1}, \ldots, x_{5} \in V$,

$$
\begin{aligned}
m\left(m\left(x_{1} \otimes x_{2} \otimes x_{3}\right) \otimes x_{4} \otimes x_{5}\right) & =m\left(x_{1} \otimes m\left(x_{2} \otimes x_{3} \otimes x_{4}\right) \otimes x_{5}\right) \\
& =m\left(x_{1} \otimes x_{2} \otimes m\left(x_{3} \otimes x_{4} \otimes x_{5}\right)\right)
\end{aligned}
$$

Example 2.2. Let $\left\{e_{1}, e_{2}\right\}$ be a basis of a 2-dimensional space $V=\mathbb{K}^{2}$, the ternary operation on $V$ given by

$$
\begin{array}{ll}
m\left(e_{1} \otimes e_{1} \otimes e_{1}\right)=e_{1}, & m\left(e_{2} \otimes e_{2} \otimes e_{1}\right)=e_{1}+e_{2} \\
m\left(e_{1} \otimes e_{1} \otimes e_{2}\right)=e_{2}, & m\left(e_{2} \otimes e_{2} \otimes e_{2}\right)=e_{1}+2 e_{2} \\
m\left(e_{1} \otimes e_{2} \otimes e_{2}\right)=e_{1}+e_{2}, & m\left(e_{1} \otimes e_{2} \otimes e_{1}\right)=e_{2} \\
m\left(e_{2} \otimes e_{1} \otimes e_{1}\right)=e_{2}, & m\left(e_{2} \otimes e_{1} \otimes e_{2}\right)=e_{1}+e_{2}
\end{array}
$$

defines a totally associative ternary algebra.

Definition 2.3. A weak totally associative ternary algebra is given by a $\mathbb{K}$-vector space $V$ and a ternary operation $m$, satisfying for every $x_{1}, \ldots, x_{5} \in V$,

$$
m\left(m\left(x_{1} \otimes x_{2} \otimes x_{3}\right) \otimes x_{4} \otimes x_{5}\right)=m\left(x_{1} \otimes x_{2} \otimes m\left(x_{3} \otimes x_{4} \otimes x_{5}\right)\right)
$$

Naturally, every totally associative ternary algebra is a weak totally associative ternary algebra.

Definition 2.4. A partially associative ternary algebra is given by a $\mathbb{K}$-vector space $V$ and a ternary operation $m$ satisfying, for every $x_{1}, \ldots, x_{5} \in V$,

$$
\begin{aligned}
& m\left(m\left(x_{1} \otimes x_{2} \otimes x_{3}\right) \otimes x_{4} \otimes x_{5}\right)+m\left(x_{1} \otimes m\left(x_{2} \otimes x_{3} \otimes x_{4}\right) \otimes x_{5}\right) \\
& \quad+m\left(x_{1} \otimes x_{2} \otimes m\left(x_{3} \otimes x_{4} \otimes x_{5}\right)\right)=0
\end{aligned}
$$

Example 2.5. Let $\left\{e_{1}, e_{2}\right\}$ be a basis of a 2-dimensional space $V=\mathbb{K}^{2}$, the ternary operation on $V$ given by $m\left(e_{1} \otimes e_{1} \otimes e_{1}\right)=e_{2}$ defines a partially associative ternary algebra.

We introduce in the following some variants of partial total associativity of ternary operations. 
Definition 2.6. An alternate partially associative ternary algebra of first kind is given by a $\mathbb{K}$-vector space $V$ and a ternary operation $m$ satisfying, for every $x_{1}, \ldots, x_{5} \in V$,

$$
\begin{aligned}
& m\left(m\left(x_{1} \otimes x_{2} \otimes x_{3}\right) \otimes x_{4} \otimes x_{5}\right)-m\left(x_{1} \otimes m\left(x_{2} \otimes x_{3} \otimes x_{4}\right) \otimes x_{5}\right) \\
& \quad+m\left(x_{1} \otimes x_{2} \otimes m\left(x_{3} \otimes x_{4} \otimes x_{5}\right)\right)=0
\end{aligned}
$$

The alternate partially associative ternary algebra is of second kind it satisfies

$$
\begin{aligned}
& m\left(m\left(x_{1} \otimes x_{2} \otimes x_{3}\right) \otimes x_{4} \otimes x_{5}\right)-m\left(x_{1} \otimes m\left(x_{2} \otimes x_{3} \otimes x_{4}\right) \otimes x_{5}\right) \\
& \quad-m\left(x_{1} \otimes x_{2} \otimes m\left(x_{3} \otimes x_{4} \otimes x_{5}\right)\right)=0
\end{aligned}
$$

Remark 2.7. Let $(V, \cdot)$ be a bilinear associative algebra. Then, the ternary operation, defined by $m\left(x_{1} \otimes x_{2} \otimes x_{3}\right)=\left(x_{1} \cdot x_{2} \cdot x_{3}\right)$, determines on the vector space $V$ a structure of totally associative ternary algebra which is not partially associative.

Definition 2.8. A ternary operation $m$ is said to be commutative if

$$
\sum_{\sigma \in S_{3}} \operatorname{Sgn}(\sigma) m\left(x_{\sigma(1)} \otimes x_{\sigma(2)} \otimes x_{\sigma(3)}\right)=0, \quad \forall x_{1}, x_{2}, x_{3} \in V
$$

Remark 2.9. A symmetric ternary operation is commutative.

In the following, we recall the definitions of ternary algebras of Lie type.

Definition 2.10. A ternary Lie algebras is a skew-symmetric ternary operation $[$, , ] on a $\mathbb{K}$-vector space $V$ satisfying $\forall x_{1}, \ldots, x_{5} \in V$ the following generalized Jacobi condition:

$$
\sum_{\sigma \in S_{3}} \operatorname{Sgn}(\sigma)\left[\left[x_{\sigma\left(x_{1}\right)}, x_{\sigma\left(x_{2}\right)}, x_{\sigma\left(x_{3}\right)}\right], x_{\sigma\left(x_{4}\right)}, x_{\sigma\left(x_{5}\right)}\right]=0
$$

As in the binary case, there is a functor which makes correspondence to any partially associative ternary algebra a ternary Lie algebra (see [20, 21]).

Proposition 2.11. To any partially associative ternary algebra on a vector space $V$ with ternary operation $m$, one associates a ternary Lie algebra on $V$ defined $\forall x_{1}, x_{2}, x_{3} \in V$ by the bracket

$$
\left[x_{1}, x_{2}, x_{3}\right]=\sum_{\sigma \in S_{3}} \operatorname{Sgn} m\left(x_{\sigma(1)} \otimes x_{\sigma(2)} \otimes x_{\sigma(3)}\right)
$$

There is another kind of ternary algebras of Lie type, they are called ternary Nambu algebra. They appeared naturally in Nambu mechanics which is a generalization of classical mechanics.

Definition 2.12. A ternary Nambu algebra is a ternary bracket on a $\mathbb{K}$-vector space $V$ satisfying a so-called fundamental or Filippov identity:

$$
\begin{aligned}
{\left[x_{1}, x_{2},\left[x_{3}, x_{4}, x_{5}\right]\right]=} & {\left[\left[x_{1}, x_{2}, x_{3}\right], x_{4}, x_{5}\right]+\left[x_{3},\left[x_{1}, x_{2}, x_{4}\right], x_{5}\right] } \\
& +\left[x_{3}, x_{4},\left[x_{1}, x_{2}, x_{5}\right]\right], \quad \forall x_{1}, \ldots, x_{5} \in V
\end{aligned}
$$

When the bracket is skew-symmetric, the ternary algebra is called ternary Nambu-Lie algebra.

The Lie triple system is defined as a vector space $V$ equipped with a ternary bracket that satisfies the same fundamental identity as a Nambu-Lie bracket but instead of skewsymmetry, it satisfies the condition

$$
\left[x_{1}, x_{2}, x_{3}\right]+\left[x_{2}, x_{3}, x_{1}\right]+\left[x_{3}, x_{1}, x_{2}\right]=0
$$


Example 2.13. The polynomial algebra of 3 variables $x_{1}, x_{2}, x_{3}$, endowed with a ternary operation defined by the functional Jacobian:

$$
\left[f_{1}, f_{2}, f_{3}\right]=\left|\begin{array}{lll}
\frac{\delta f_{1}}{\delta x_{1}} & \frac{\delta f_{1}}{\delta x_{2}} & \frac{\delta f_{1}}{\delta x_{3}} \\
\frac{\delta f_{2}}{\delta x_{1}} & \frac{\delta f_{2}}{\delta x_{2}} & \frac{\delta f_{2}}{\delta x_{3}} \\
\frac{\delta f_{3}}{\delta x_{1}} & \frac{\delta f_{3}}{\delta x_{2}} & \frac{\delta f_{3}}{\delta x_{3}}
\end{array}\right|
$$

is a ternary Nambu-Lie algebra.

We have also the following fundamental example.

Example 2.14. Let $V=\mathbb{R}^{4}$ be the 4-dimensional oriented Euclidian space over $\mathbb{R}$. The bracket of 3 vectors $\overrightarrow{x_{1}}, \overrightarrow{x_{2}}, \overrightarrow{x_{3}}$ is given by

$$
\left[\overrightarrow{x_{1}}, \overrightarrow{x_{2}}, \overrightarrow{x_{3}}\right]=\overrightarrow{x_{1}} \times \overrightarrow{x_{2}} \times \overrightarrow{x_{3}}=\left|\begin{array}{cccc}
x_{11} & x_{12} & x_{13} & \overrightarrow{e_{1}} \\
x_{21} & x_{22} & x_{23} & \overrightarrow{e_{2}} \\
x_{31} & x_{32} & x_{33} & \overrightarrow{e_{3}} \\
x_{41} & x_{42} & x_{43} & \overrightarrow{e_{4}}
\end{array}\right|
$$

where $\left(x_{1 r}, \ldots, x_{4 r}\right)_{r=1,2,3}$ are the coordinates of $\overrightarrow{x_{r}}$ with respect to orthonormal basis $\left\{e_{r}\right\}$. Then, $(V,[\cdot, \cdot, \cdot])$ is a ternary Nambu-Lie algebra.

Remark 2.15. Every ternary Nambu-Lie algebra on $\mathbb{R}^{4}$ could be deduced from the previous example (see [14]).

\subsection{Homological algebra of ternary algebras}

The basic concepts of homological algebra are those of a complex and homomorphisms of complexes, defining the category of complexes (see, e.g., [58]). A chain complex $\mathcal{C}$. is a sequence $\mathcal{C}=\left\{\mathcal{C}_{p}\right\}_{p}$ of abelian groups or more generally objects of an abelian category and an indexed set $\delta=\left\{\delta_{p}\right\}_{p}$ of homomorphisms $\delta_{p}: \mathcal{C}_{p} \rightarrow \mathcal{C}_{p-1}$ such that $\delta_{p-1} \circ \delta_{p}=0$ for all $p$. A chain complex can be considered as a cochain complex by reversing the enumeration $\mathcal{C}^{p}=\mathcal{C}_{-p}$ and $\delta^{p}=\delta_{-p}$. A cochain complex $\mathcal{C}$ is a sequence of abelian groups and homomorphisms $\cdots \stackrel{\delta^{p-1}}{\longrightarrow} \mathcal{C}^{p} \stackrel{\delta^{p}}{\longrightarrow} \mathcal{C}^{p+1} \stackrel{\delta^{p+1}}{\longrightarrow} \cdots$ with the property $\delta^{p+1} \circ \delta^{p}=0$ for all $p$.

The homomorphisms $\delta^{p}$ are called coboundary operators or codifferentials. A cohomology of a cochain complex $\mathcal{C}$ is given by the groups $H^{p}(\mathcal{C})=\operatorname{Ker} \delta^{p} / \operatorname{Im} \delta^{p-1}$.

The elements of $\mathcal{C}^{p}$ are $p$-cochains, the elements of $Z^{p}:=\operatorname{Ker} \delta^{p}$ are $p$-cocycles, and the elements of $B^{p}:=\operatorname{Im} \delta^{p-1}$ are $p$-coboundaries. Because $\delta^{p+1} \circ \delta^{p}=0$ for all $p$, we have $0 \subseteq B^{p} \subseteq Z^{p} \subseteq \mathcal{C}^{p}$ for all $p$. The $p$ th cohomology group is the quotient $H^{p}=Z^{p} / B^{p}$.

We introduce in the following the $p$-cochains for a ternary algebra of associative type $\mathcal{A}=(V, m)$.

Definition 2.16. We call $p$-cochain of a ternary algebra $\mathcal{A}=(V, m)$ a linear map $\varphi$ : $V^{\otimes 2 p+1} \longrightarrow V$. The $p$-cochains set on $V$ is

$$
\mathcal{C}^{p}(\mathcal{A}, \mathcal{A})=\{\varphi: V^{\otimes 2 p+1}=\underbrace{V \otimes V \otimes \cdots \otimes V}_{2 p+1 \text { times }} \longrightarrow V\}
$$


Remark 2.17. The set $\mathcal{C}^{p}(\mathcal{A}, \mathcal{A})$ is an abelian group.

We define a circle operation on cochains as usual, that is, a map

$$
\circ: \mathcal{C}^{p}(\mathcal{A}, \mathcal{A}) \times \mathcal{C}^{q}(\mathcal{A}, \mathcal{A}) \longrightarrow \mathcal{C}^{p+q}(\mathcal{A}, \mathcal{A}) \quad(\varphi, \psi) \longmapsto \varphi \circ \psi
$$

such that

$$
\begin{aligned}
(\varphi \circ \psi) & \left(x_{1} \otimes \cdots \otimes x_{2 p+2 q+1}\right) \\
= & \sum_{i=0}^{2 p} \varphi\left(x_{1} \otimes \cdots \otimes \psi\left(x_{i+1} \otimes \cdots \otimes x_{i+2 q+1}\right) \otimes \cdots \otimes x_{2 p+2 q+1}\right)
\end{aligned}
$$

One has a cochain complex for ternary algebras $\mathcal{A}$ with values in $\mathcal{A}$ if there exists a sequence of abelian groups and homomorphisms $\cdots \stackrel{\delta^{p-1}}{\longrightarrow} \mathcal{C}^{p}(\mathcal{A}, \mathcal{A}) \stackrel{\delta^{p}}{\longrightarrow} \mathcal{C}^{p+1}(\mathcal{A}, \mathcal{A}) \stackrel{\delta^{p+1}}{\longrightarrow} \cdots$ such that for all $p, \delta^{p+1} \circ \delta^{p}=0$.

\section{Cohomology of partially associative ternary algebras}

We have studied in [2] deformations of partially associative ternary algebras which are intimately linked to cohomology groups. We have introduced the operators $\delta^{1}$ and $\delta^{2}$ which should correspond to a complex of partially associative ternary algebra defining a deformation cohomology. In the following, we recall the definitions of $\delta^{1}$ and $\delta^{2}$ and show that it is impossible to extend these operators to a usual operator $\delta^{3}$. As a consequence, we deduce that the operad of partially associative ternary algebras is not Koszul, see [45, 19] about Koszulity.

Let $\mathcal{A}=(V, m)$ be a partially associative ternary algebra on a $\mathbb{K}$-vector space $V$.

Definition 3.1. We call ternary 1-coboundary operator the map

$$
\delta^{1}: \mathcal{C}^{0}(\mathcal{A}, \mathcal{A}) \longrightarrow \mathcal{C}^{1}(\mathcal{A}, \mathcal{A}), \quad f \longmapsto \delta^{1} f
$$

defined by

$$
\begin{aligned}
\delta^{1} f\left(x_{1} \otimes x_{2} \otimes x_{3}\right)= & f\left(m\left(x_{1} \otimes x_{2} \otimes x_{3}\right)\right)-m\left(f\left(x_{1}\right) \otimes x_{2} \otimes x_{3}\right) \\
& -m\left(x_{1} \otimes f\left(x_{2}\right) \otimes x_{3}\right)-m\left(x_{1} \otimes x_{2} \otimes f\left(x_{3}\right)\right)
\end{aligned}
$$

Definition 3.2. We call ternary 2-coboundary operator the map

$$
\delta^{2}: \mathcal{C}^{1}(\mathcal{A}, \mathcal{A}) \longrightarrow \mathcal{C}^{2}(\mathcal{A}, \mathcal{A}), \quad \varphi \longmapsto \delta^{2} \varphi
$$

defined by

$$
\begin{aligned}
\delta^{2} \varphi & \left(x_{1} \otimes x_{2} \otimes x_{3} \otimes x_{4} \otimes x_{5}\right) \\
= & m\left(\varphi\left(x_{1} \otimes x_{2} \otimes x_{3}\right) \otimes x_{4} \otimes x_{5}\right)+m\left(x_{1} \otimes \varphi\left(x_{2} \otimes x_{3} \otimes x_{4}\right) \otimes x_{5}\right) \\
& +m\left(x_{1} \otimes x_{2} \otimes \varphi\left(x_{3} \otimes x_{4} \otimes x_{5}\right)\right)+\varphi\left(m\left(x_{1} \otimes x_{2} \otimes x_{3}\right) \otimes x_{4} \otimes x_{5}\right) \\
& +\varphi\left(x_{1} \otimes m\left(x_{2} \otimes x_{3} \otimes x_{4}\right) \otimes x_{5}\right)+\varphi\left(x_{1} \otimes x_{2} \otimes m\left(x_{3} \otimes x_{4} \otimes x_{5}\right)\right)
\end{aligned}
$$

Remark 3.3. The operator $\delta^{2}$ can also be defined by $\delta^{2} \varphi=\varphi \circ m+m \circ \varphi$. 
Proposition 3.4. We have $\delta^{2} \circ \delta^{1}=0$.

Proof. Let $f$ be a 0 -cochain. We compute $\delta^{2}\left(\delta^{1} f\right)$.

We have for all $x_{1}, x_{2}, x_{3}, x_{4}, x_{5} \in V$,

$$
\begin{aligned}
\delta^{2}( & \left.\delta^{1} f\right)\left(x_{1} \otimes x_{2} \otimes x_{3} \otimes x_{4} \otimes x_{5}\right) \\
= & m\left(f\left(m\left(x_{1} \otimes x_{2} \otimes x_{3}\right)\right) \otimes x_{4} \otimes x_{5}\right)-m\left(m\left(f\left(x_{1}\right) \otimes x_{2} \otimes x_{3}\right) \otimes x_{4} \otimes x_{5}\right) \\
& -m\left(m\left(x_{1} \otimes f\left(x_{2}\right) \otimes x_{3}\right) \otimes x_{4} \otimes x_{5}\right)-m\left(m\left(x_{1} \otimes x_{2} \otimes f\left(x_{3}\right)\right) \otimes x_{4} \otimes x_{5}\right) \\
& +m\left(x_{1} \otimes f\left(m\left(x_{2} \otimes x_{3} \otimes x_{4}\right)\right) \otimes x_{5}\right)-m\left(x_{1} \otimes m\left(f\left(x_{2}\right) \otimes x_{3} \otimes x_{4}\right) \otimes x_{5}\right) \\
& -m\left(x_{1} \otimes m\left(x_{2} \otimes f\left(x_{3}\right) \otimes x_{4}\right) \otimes x_{5}\right)-m\left(x_{1} \otimes m\left(x_{2} \otimes x_{3} \otimes f\left(x_{4}\right)\right) \otimes x_{5}\right) \\
& +m\left(x_{1} \otimes x_{2} \otimes\left(f\left(m\left(x_{3} \otimes x_{4} \otimes x_{5}\right)\right)\right)\right)-m\left(x_{1} \otimes x_{2} \otimes m\left(f\left(x_{3}\right) \otimes x_{4} \otimes x_{5}\right)\right) \\
& -m\left(x_{1} \otimes x_{2} \otimes m\left(x_{3} \otimes f\left(x_{4}\right) \otimes x_{5}\right)\right)-m\left(x_{1} \otimes x_{2} \otimes m\left(x_{3} \otimes x_{4} \otimes f\left(x_{5}\right)\right)\right) \\
& +f\left(m\left(m\left(x_{1} \otimes x_{2} \otimes x_{3}\right) \otimes x_{4} \otimes x_{5}\right)\right)-m\left(f\left(m\left(x_{1} \otimes x_{2} \otimes x_{3}\right)\right) \otimes x_{4} \otimes x_{5}\right) \\
& -m\left(m\left(x_{1} \otimes x_{2} \otimes x_{3}\right) \otimes f\left(x_{4}\right) \otimes x_{5}\right)-m\left(m\left(x_{1} \otimes x_{2} \otimes x_{3}\right) \otimes x_{4} \otimes f\left(x_{5}\right)\right) \\
& +f\left(m\left(x_{1} \otimes m\left(x_{2} \otimes x_{3} \otimes x_{4}\right) \otimes x_{5}\right)\right)-m\left(f\left(x_{1}\right) \otimes m\left(x_{2} \otimes x_{3} \otimes x_{4}\right) \otimes x_{5}\right) \\
& -m\left(x_{1} \otimes f\left(m\left(x_{2} \otimes x_{3} \otimes x_{4}\right)\right) \otimes x_{5}\right)-m\left(x_{1} \otimes m\left(x_{2} \otimes x_{3} \otimes x_{4}\right) \otimes f\left(x_{5}\right)\right) \\
& +f\left(m\left(x_{1} \otimes x_{2} \otimes m\left(x_{3} \otimes x_{4} \otimes x_{5}\right)\right)\right)-m\left(f\left(x_{1}\right) \otimes x_{2} \otimes m\left(x_{3} \otimes x_{4} \otimes x_{5}\right)\right) \\
& -m\left(x_{1} \otimes f\left(x_{2}\right) \otimes m\left(x_{3} \otimes x_{4} \otimes x_{5}\right)\right)-m\left(x_{1} \otimes x_{2} \otimes f\left(m\left(x_{3} \otimes x_{4} \otimes x_{5}\right)\right)\right)=0
\end{aligned}
$$

The cohomology spaces relative to these coboundary operators are as follows.

Definition 3.5. The 1-cocycles space of $\mathcal{A}$ is

$$
Z^{1}(\mathcal{A}, \mathcal{A})=\left\{f: V \longrightarrow V: \delta^{1} f=0\right\}
$$

The 2-coboundaries space of $\mathcal{A}$ is

$$
B^{2}(\mathcal{A}, \mathcal{A})=\left\{\varphi: V^{\otimes 3} \longrightarrow V: \varphi=\delta^{1} f, f \in \mathcal{C}^{0}(\mathcal{A}, \mathcal{A})\right\}
$$

The 2-cocycles space of $\mathcal{A}$ is

$$
Z^{2}(\mathcal{A}, \mathcal{A})=\left\{f: V^{\otimes 3} \longrightarrow V: \delta^{2} f=0\right\}
$$

Remark 3.6. One has $B^{2}(\mathcal{A}, \mathcal{A}) \subset Z^{2}(\mathcal{A}, \mathcal{A})$, because $\delta^{2} \circ \delta^{1}=0$. Note also that $Z^{1}(\mathcal{A}, \mathcal{A})$ corresponds to the derivations space, denoted also by $\operatorname{Der}(\mathcal{A})$, of the partially associative ternary algebra $\mathcal{A}$.

Definition 3.7. We call the $p$ th cohomology group $(p=0,1)$ of the partially associative ternary algebra $\mathcal{A}$ the quotient

$$
H^{p}(\mathcal{A}, \mathcal{A})=\frac{Z^{p}(\mathcal{A}, \mathcal{A})}{B^{p}(\mathcal{A}, \mathcal{A})}, \quad p=1,2
$$

The following proposition shows that we cannot extend the 1-cohomology and 2-cohomology corresponding to the operators $\delta^{1}$ and $\delta^{2}$ to a usual 3-cohomology. 
Proposition 3.8. Let $\mathcal{A}=(V, m)$ be a partially associative ternary algebra. There is no usual 3-cohomology extending the 2-cohomology corresponding to the coboundary operator

$$
\delta^{2}: \mathcal{C}^{1}(\mathcal{A}, \mathcal{A}) \longrightarrow \mathcal{C}^{2}(\mathcal{A}, \mathcal{A})
$$

defined for all $x_{1}, x_{2}, x_{3}, x_{4}, x_{5} \in V$ by

$$
\begin{aligned}
\delta^{2} \varphi & \left(x_{1} \otimes x_{2} \otimes x_{3} \otimes x_{4} \otimes x_{5}\right) \\
= & m\left(\varphi\left(x_{1} \otimes x_{2} \otimes x_{3}\right) \otimes x_{4} \otimes x_{5}\right)+m\left(x_{1} \otimes \varphi\left(x_{2} \otimes x_{3} \otimes x_{4}\right) \otimes x_{5}\right) \\
& +m\left(x_{1} \otimes x_{2} \otimes \varphi\left(x_{3} \otimes x_{4} \otimes x_{5}\right)\right)+\varphi\left(m\left(x_{1} \otimes x_{2} \otimes x_{3}\right) \otimes x_{4} \otimes x_{5}\right) \\
& +\varphi\left(x_{1} \otimes m\left(x_{2} \otimes x_{3} \otimes x_{4}\right) \otimes x_{5}\right)+\varphi\left(x_{1} \otimes x_{2} \otimes m\left(x_{3} \otimes x_{4} \otimes x_{5}\right)\right)
\end{aligned}
$$

Proof. We consider a 3 -cochain $f$, that is, a map $f: V^{\otimes 5} \rightarrow V$, and set

$$
\begin{aligned}
& \delta^{3} f\left(x_{1} \otimes x_{2} \otimes x_{3} \otimes x_{4} \otimes x_{5} \otimes x_{6} \otimes x_{7}\right) \\
&=a_{1} m\left(x_{1} \otimes x_{2} \otimes f\left(x_{3} \otimes x_{4} \otimes x_{5} \otimes x_{6} \otimes x_{7}\right)\right) \\
&+a_{2} m\left(x_{1} \otimes f\left(x_{2} \otimes x_{3} \otimes x_{4} \otimes x_{5} \otimes x_{6}\right) \otimes x_{7}\right) \\
&+a_{3} m\left(f\left(x_{1} \otimes x_{2} \otimes x_{3} \otimes x_{4} \otimes x_{5}\right) \otimes x_{6} \otimes x_{7}\right) \\
&+a_{4} f\left(m\left(x_{1} \otimes x_{2} \otimes x_{3}\right) \otimes x_{4} \otimes x_{5} \otimes x_{6} \otimes x_{7}\right) \\
&+a_{5} f\left(x_{1} \otimes m\left(x_{2} \otimes x_{3} \otimes x_{4}\right) \otimes x_{5} \otimes x_{6} \otimes x_{7}\right) \\
&+a_{6} f\left(x_{1} \otimes x_{2} \otimes m\left(x_{3} \otimes x_{4} \otimes x_{5}\right) \otimes x_{6} \otimes x_{7}\right) \\
&+a_{7} f\left(x_{1} \otimes x_{2} \otimes x_{3} \otimes m\left(x_{4} \otimes x_{5} \otimes x_{6}\right) \otimes x_{7}\right) \\
&+a_{8} f\left(x_{1} \otimes x_{2} \otimes x_{3} \otimes x_{4} \otimes m\left(x_{5} \otimes x_{6} \otimes x_{7}\right)\right)
\end{aligned}
$$

where $a_{1}, \ldots, a_{8} \in \mathbb{K}$.

Let $g$ be a 2-cochain, that is, a map $f: V^{\otimes 5} \rightarrow V$. We compute $\delta^{3}\left(\delta^{2} g\right)\left(x_{1} \otimes x_{2} \otimes x_{3} \otimes\right.$ $\left.x_{4} \otimes x_{5} \otimes x_{6} \otimes x_{7}\right)$ and substitute $m\left(y_{1} \otimes y_{2} \otimes m\left(y_{3} \otimes y_{4} \otimes y_{5}\right)\right)$ by

$$
-m\left(y_{1} \otimes m\left(y_{2} \otimes y_{3} \otimes y_{4}\right) \otimes y_{5}\right)-m\left(m\left(y_{1} \otimes y_{2} \otimes y_{3}\right) \otimes y_{4} \otimes y_{5}\right)
$$

Then, we obtain

$$
\begin{aligned}
\delta^{3}\left(\delta^{2} g\right) & \left(x_{1} \otimes x_{2} \otimes x_{3} \otimes x_{4} \otimes x_{5} \otimes x_{6} \otimes x_{7}\right) \\
= & \left(a_{7}-a_{8}\right) g\left(x_{1} \otimes x_{2} \otimes m\left(x_{3} \otimes m\left(x_{4} \otimes x_{5} \otimes x_{6}\right) \otimes x_{7}\right)\right) \\
& +\left(a_{6}-a_{8}\right) g\left(x_{1} \otimes x_{2} \otimes m\left(m\left(x_{3} \otimes x_{4} \otimes x_{5}\right) \otimes x_{6} \otimes x_{7}\right)\right) \\
& +\left(a_{5}+a_{8}\right) g\left(x_{1} \otimes m\left(x_{2} \otimes x_{3} \otimes x_{4}\right) \otimes m\left(x_{5} \otimes x_{6} \otimes x_{7}\right)\right) \\
& +\left(a_{6}-a_{7}\right) g\left(x_{1} \otimes m\left(x_{2} \otimes m\left(x_{3} \otimes x_{4} \otimes x_{5}\right) \otimes x_{6}\right) \otimes x_{7}\right) \\
& +\left(a_{5}-a_{7}\right) g\left(x_{1} \otimes m\left(m\left(x_{2} \otimes x_{3} \otimes x_{4}\right) \otimes x_{5} \otimes x_{6}\right) \otimes x_{7}\right) \\
& +\left(a_{4}+a_{8}\right) g\left(m\left(x_{1} \otimes x_{2} \otimes x_{3}\right) \otimes x_{4} \otimes m\left(x_{5} \otimes x_{6} \otimes x_{7}\right)\right) \\
& +\left(a_{4}+a_{7}\right) g\left(m\left(x_{1} \otimes x_{2} \otimes x_{3}\right) \otimes m\left(x_{4} \otimes x_{5} \otimes x_{6}\right) \otimes x_{7}\right) \\
& +\left(a_{5}-a_{6}\right) g\left(m\left(x_{1} \otimes m\left(x_{2} \otimes x_{3} \otimes x_{4}\right) \otimes x_{5}\right) \otimes x_{6} \otimes x_{7}\right) \\
& +\left(a_{4}-a_{6}\right) g\left(m\left(m\left(x_{1} \otimes x_{2} \otimes x_{3}\right) \otimes x_{4} \otimes x_{5}\right) \otimes x_{6} \otimes x_{7}\right) \\
& +\left(a_{1}+a_{8}\right) m\left(x_{1} \otimes x_{2} \otimes g\left(x_{3} \otimes x_{4} \otimes m\left(x_{5} \otimes x_{6} \otimes x_{7}\right)\right)\right)
\end{aligned}
$$




$$
\begin{aligned}
& +\left(a_{1}+a_{7}\right) m\left(x_{1} \otimes x_{2} \otimes g\left(x_{3} \otimes m\left(x_{4} \otimes x_{5} \otimes x_{6}\right) \otimes x_{7}\right)\right) \\
& +\left(a_{1}+a_{6}\right) m\left(x_{1} \otimes x_{2} \otimes g\left(m\left(x_{3} \otimes x_{4} \otimes x_{5}\right) \otimes x_{6} \otimes x_{7}\right)\right) \\
& +\left(a_{2}+a_{7}\right) m\left(x_{1} \otimes g\left(x_{2} \otimes x_{3} \otimes m\left(x_{4} \otimes x_{5} \otimes x_{6}\right)\right) \otimes x_{7}\right) \\
& +\left(a_{2}+a_{6}\right) m\left(x_{1} \otimes g\left(x_{2} \otimes m\left(x_{3} \otimes x_{4} \otimes x_{5}\right) \otimes x_{6}\right) \otimes x_{7}\right) \\
& +\left(a_{2}+a_{5}\right) m\left(x_{1} \otimes g\left(m\left(x_{2} \otimes x_{3} \otimes x_{4}\right) \otimes x_{5} \otimes x_{6}\right) \otimes x_{7}\right) \\
& +\left(a_{5}-a_{1}\right) m\left(x_{1} \otimes m\left(x_{2} \otimes x_{3} \otimes x_{4}\right) \otimes g\left(x_{5} \otimes x_{6} \otimes x_{7}\right)\right) \\
& +\left(a_{2}-a_{1}\right) m\left(x_{1} \otimes m\left(x_{2} \otimes x_{3} \otimes g\left(x_{4} \otimes x_{5} \otimes x_{6}\right)\right) \otimes x_{7}\right) \\
& +\left(a_{2}-a_{1}\right) m\left(x_{1} \otimes m\left(x_{2} \otimes g\left(x_{3} \otimes x_{4} \otimes x_{5}\right) \otimes x_{6}\right) \otimes x_{7}\right) \\
& +\left(a_{2}-a_{8}\right) m\left(x_{1} \otimes m\left(g\left(x_{2} \otimes x_{3} \otimes x_{4}\right) \otimes x_{5} \otimes x_{6}\right) \otimes x_{7}\right) \\
& +\left(a_{7}-a_{8}\right) m\left(g\left(x_{1} \otimes x_{2} \otimes x_{3}\right) \otimes m\left(x_{4} \otimes x_{5} \otimes x_{6}\right) \otimes x_{7}\right) \\
& +\left(a_{3}+a_{6}\right) m\left(g\left(x_{1} \otimes x_{2} \otimes m\left(x_{3} \otimes x_{4} \otimes x_{5}\right)\right) \otimes x_{6} \otimes x_{7}\right) \\
& +\left(a_{3}+a_{5}\right) m\left(g\left(x_{1} \otimes m\left(x_{2} \otimes x_{3} \otimes x_{4}\right) \otimes x_{5}\right) \otimes x_{6} \otimes x_{7}\right) \\
& +\left(a_{3}+a_{4}\right) m\left(g\left(m\left(x_{1} \otimes x_{2} \otimes x_{3}\right) \otimes x_{4} \otimes x_{5}\right) \otimes x_{6} \otimes x_{7}\right) \\
& +\left(a_{4}-a_{1}\right) m\left(m\left(x_{1} \otimes x_{2} \otimes x_{3}\right) \otimes x_{4} \otimes g\left(x_{5} \otimes x_{6} \otimes x_{7}\right)\right) \\
& +\left(a_{4}-a_{1}\right) m\left(m\left(x_{1} \otimes x_{2} \otimes x_{3}\right) \otimes g\left(x_{4} \otimes x_{5} \otimes x_{6}\right) \otimes x_{7}\right) \\
& +\left(a_{3}-a_{1}\right) m\left(m\left(x_{1} \otimes x_{2} \otimes g\left(x_{3} \otimes x_{4} \otimes x_{5}\right)\right) \otimes x_{6} \otimes x_{7}\right) \\
& +\left(a_{3}-a_{8}\right) m\left(m\left(x_{1} \otimes g\left(x_{2} \otimes x_{3} \otimes x_{4}\right) \otimes x_{5}\right) \otimes x_{6} \otimes x_{7}\right) \\
& +\left(a_{3}-a_{8}\right) m\left(m\left(g\left(x_{1} \otimes x_{2} \otimes x_{3}\right) \otimes x_{4} \otimes x_{5}\right) \otimes x_{6} \otimes x_{7}\right)=0
\end{aligned}
$$

The equation is satisfied for all $x_{1}, x_{2}, x_{3}, x_{4}, x_{5}, x_{6}, x_{7} \in V$ if and only if $a_{1}, \ldots, a_{8}$ are all equal to 0.

Corollary 3.9. A usual deformation cohomology of partially associative ternary algebras does not exist. Then, the operad of the partially associative ternary algebras $p A s s^{(3)}$ is not a Koszul operad.

Remark 3.10. In [28], it is shown that the operad of totally associative ternary algebras is Koszul because it has a Poincaré-Birkhoff-Witt basis. Moreover, its dual, the operad of partially associative ternary algebras, is also Koszul when the operations are in degree one. See also [22] for constructions in this case and the recent preprint [53]. The corollary claims that the operad is not a Koszul operad when the operations are in degree zero.

Remark 3.11. Using the same approach, we can show that the alternate partially associative ternary algebras of first and second kind do not carry a usual deformation cohomology as well, then their operads are not koszul operads.

\section{Cohomology of weak totally associative ternary algebras}

In this section, we generalize to $p$-cohomology, for all $p$, the 1-cohomology and 2-cohomology of weak totally associative ternary algebra defined by Takhtajan (see [56]). Let $\mathcal{A}=(V, m)$ be a weak totally associative ternary algebras on a $\mathbb{K}$-vector space $V$.

The 1-coboundary and 2-coboundary operators for weak totally associative ternary algebras $\mathcal{A}$ are defined as follows 
Definition 4.1. A 1-coboundary operator of a weak totally associative ternary algebra $\mathcal{A}=(V, m)$ is the map

$$
\delta^{1}: \mathcal{C}^{1}(\mathcal{A}, \mathcal{A}) \longrightarrow \mathcal{C}^{2}(\mathcal{A}, \mathcal{A}), \quad f \longmapsto \delta^{1} f
$$

defined for all $x_{1}, x_{2}, x_{3} \in V$ by

$$
\begin{aligned}
\delta^{1} f\left(x_{1} \otimes x_{2} \otimes x_{3}\right)= & m\left(f\left(x_{1}\right) \otimes x_{2} \otimes x_{3}\right)+m\left(x_{1} \otimes f\left(x_{2}\right) \otimes x_{3}\right) \\
& +m\left(x_{1} \otimes x_{2} \otimes f\left(x_{3}\right)\right)-f\left(m\left(x_{1} \otimes x_{2} \otimes x_{3}\right)\right)
\end{aligned}
$$

A 2-coboundary operator of a weak totally associative ternary algebra $\mathcal{A}$ is the map

$$
\begin{aligned}
\delta^{2}: \mathcal{C}^{2}(\mathcal{A}, \mathcal{A}) & \longrightarrow \mathcal{C}^{3}(\mathcal{A}, \mathcal{A}) \\
\varphi & \longmapsto \delta^{2} \varphi
\end{aligned}
$$

defined for all $x_{1}, \ldots, x_{5} \in V$ by

$$
\begin{aligned}
& \delta^{2} \varphi\left(x_{1} \otimes x_{2} \otimes x_{3} \otimes x_{4} \otimes x_{5}\right) \\
& \quad=m\left(x_{1} \otimes x_{2} \otimes \varphi\left(x_{3} \otimes x_{4} \otimes x_{5}\right)\right)-m\left(\varphi\left(x_{1} \otimes x_{2} \otimes x_{3}\right) \otimes x_{4} \otimes x_{5}\right) \\
& \quad+\varphi\left(x_{1} \otimes x_{2} \otimes m\left(x_{3} \otimes x_{4} \otimes x_{5}\right)\right)-\varphi\left(m\left(x_{1} \otimes x_{2} \otimes x_{3}\right) \otimes x_{4} \otimes x_{5}\right)
\end{aligned}
$$

Remark 4.2. One can easily show that $\delta^{2} \circ \delta^{1}=0$. Indeed,

$$
\begin{aligned}
\delta^{2} \circ \delta^{1} & (f)\left(x_{1} \otimes x_{2} \otimes x_{3} \otimes x_{4} \otimes x_{5}\right) \\
= & m\left(x_{1} \otimes x_{2} \otimes m\left(f\left(x_{3}\right) \otimes x_{4} \otimes x_{5}\right)\right)+m\left(x_{1} \otimes x_{2} \otimes m\left(x_{3} \otimes f\left(x_{4}\right) \otimes x_{5}\right)\right) \\
& +m\left(x_{1} \otimes x_{2} \otimes m\left(x_{3} \otimes x_{4} \otimes f\left(x_{5}\right)\right)\right)-m\left(x_{1} \otimes f\left(x_{2}\right) \otimes m\left(x_{3} \otimes x_{4} \otimes x_{5}\right)\right) \\
& -m\left(x_{1} \otimes f\left(m\left(x_{2} \otimes x_{3} \otimes x_{4}\right)\right) \otimes x_{5}\right)-m\left(m\left(x_{1} \otimes x_{2} \otimes x_{3}\right) \otimes f\left(x_{4}\right) \otimes x_{5}\right) \\
& -m\left(m\left(x_{1} \otimes x_{2} \otimes x_{3}\right) \otimes x_{4} \otimes f\left(x_{5}\right)\right)+f\left(m\left(m\left(x_{1} \otimes x_{2} \otimes x_{3}\right) \otimes x_{4} \otimes x_{5}\right)\right) \\
& +m\left(f\left(x_{1}\right) \otimes x_{2} \otimes m\left(x_{3} \otimes x_{4} \otimes x_{5}\right)\right)+m\left(x_{1} \otimes f\left(x_{2}\right) \otimes m\left(x_{3} \otimes x_{4} \otimes x_{5}\right)\right) \\
& +m\left(x_{1} \otimes x_{2} \otimes f\left(m\left(x_{3} \otimes x_{4} \otimes x_{5}\right)\right)\right)-f\left(m\left(x_{1} \otimes x_{2} \otimes m\left(x_{3} \otimes x_{4} \otimes x_{5}\right)\right)\right) \\
& -m\left(m\left(f\left(x_{1}\right) \otimes x_{2} \otimes x_{3}\right) \otimes x_{4} \otimes x_{5}\right)-m\left(m\left(x_{1} \otimes f\left(x_{2}\right) \otimes x_{3}\right) \otimes x_{4} \otimes x_{5}\right) \\
& \left.-m\left(m\left(x_{1} \otimes x_{2} \otimes f\left(x_{3}\right)\right) \otimes x_{4} \otimes x_{5}\right)+m\left(f\left(x_{1} \otimes x_{2} \otimes x_{3}\right)\right) \otimes x_{4} \otimes x_{5}\right) \\
& +f\left(m\left(x_{1} \otimes m\left(x_{2} \otimes x_{3} \otimes x_{4}\right) \otimes x_{5}\right)\right)-m\left(x_{1} \otimes m\left(x_{2} \otimes x_{3} \otimes f\left(x_{4}\right)\right) \otimes x_{5}\right) \\
& -m\left(x_{1} \otimes m\left(x_{2} \otimes x_{3} \otimes x_{4}\right) \otimes f\left(x_{5}\right)\right)+m\left(x_{1} \otimes m\left(x_{2} \otimes f\left(x_{3}\right) \otimes x_{4}\right) \otimes x_{5}\right) \\
& -m\left(x_{1} \otimes m\left(f\left(x_{2}\right) \otimes x_{3} \otimes x_{4}\right) \otimes x_{5}\right)-m\left(f\left(x_{1}\right) \otimes m\left(x_{2} \otimes x_{3} \otimes x_{4}\right) \otimes x_{5}\right)=0
\end{aligned}
$$

We introduce for weak associative ternary algebras the following generalized coboundary map.

Definition 4.3. Let $f$ be a $(p-1)$-cochain of a weak associative ternary algebra $\mathcal{A}=(V, m)$ and $f \in \mathcal{C}^{p-1}(\mathcal{A}, \mathcal{A})$, that is, $f: V^{\otimes 2 p-1} \longrightarrow V$. We set

$$
\begin{aligned}
\delta^{p} f\left(x_{1} \otimes \cdots \otimes x_{2 p+1}\right)= & m\left(x_{1} \otimes x_{2} \otimes f\left(x_{3} \otimes \cdots \otimes x_{2 p+1}\right)\right) \\
& +\sum_{i=1}^{p}(-1)^{i} f\left(x_{1} \otimes \cdots \otimes m\left(x_{2 i-1} \otimes x_{2 i} \otimes x_{2 i+1}\right) \otimes \cdots \otimes x_{2 p+1}\right) \\
& +(-1)^{p+1} m\left(f\left(x_{1} \otimes \cdots \otimes x_{2 p-1}\right) \otimes x_{2 p} \otimes x_{2 p+1}\right)
\end{aligned}
$$


In particular, we have

$$
\begin{aligned}
\delta^{3} \varphi & \left(x_{1} \otimes x_{2} \otimes x_{3} \otimes x_{4} \otimes x_{5} \otimes x_{6} \otimes x_{7}\right) \\
= & m\left(\varphi\left(x_{1} \otimes x_{2} \otimes x_{3} \otimes x_{4} \otimes x_{5}\right) \otimes x_{6} \otimes x_{7}\right) \\
& -\varphi\left(m\left(x_{1} \otimes x_{2} \otimes x_{3}\right) \otimes x_{4} \otimes x_{5} \otimes x_{6} \otimes x_{7}\right) \\
& +\varphi\left(x_{1} \otimes x_{2} \otimes m\left(x_{3} \otimes x_{4} \otimes x_{5}\right) \otimes x_{6} \otimes x_{7}\right) \\
& -\varphi\left(x_{1} \otimes x_{2} \otimes x_{3} \otimes x_{4} \otimes m\left(x_{5} \otimes x_{6} \otimes x_{7}\right)\right) \\
& +m\left(x_{1} \otimes x_{2} \otimes \varphi\left(x_{3} \otimes x_{4} \otimes x_{5} \otimes x_{6} \otimes x_{7}\right)\right)
\end{aligned}
$$

Proposition 4.4. We have $\delta^{p+1} \circ \delta^{p}=0$ for all $p \geq 1$.

Proof. We have $\delta^{2} \circ \delta^{1}=0$. Assume $\delta^{p} \circ \delta^{p-1}=0$. We have to show that $\delta^{p+1} \circ \delta^{p}=0$.

Let $\varphi$ be a $p$-cochain and $x_{1}, \ldots, x_{2 p+3} \in V$.

$$
\begin{aligned}
\delta^{p} \varphi & \left(x_{1} \otimes \cdots \otimes x_{2 p+1}\right) \\
= & m\left(x_{1} \otimes x_{2} \otimes \varphi\left(x_{3} \otimes \cdots \otimes x_{2 p+1}\right)\right) \\
& +(-1)^{p+1} m\left(\varphi\left(x_{1} \otimes \cdots \otimes x_{2 p-1}\right) \otimes x_{2 p} \otimes x_{2 p+1}\right) \\
& +\sum_{i=1}^{p}(-1)^{i} \varphi\left(x_{1} \otimes \cdots \otimes m\left(x_{2 i-1} \otimes x_{2 i} \otimes x_{2 i+1}\right) \otimes \cdots \otimes x_{2 p+1}\right)
\end{aligned}
$$

Then $\delta^{p+1}\left(\delta^{p} \varphi\right)\left(x_{1} \otimes \cdots \otimes x_{2 p+3}\right)$ vanishes. Really,

$$
\begin{aligned}
\delta^{p+1} & \left(\delta^{p} \varphi\right)\left(x_{1} \otimes \cdots \otimes x_{2 p+3}\right) \\
= & m\left(x_{1} \otimes x_{2} \otimes \delta^{p} \varphi\left(x_{3} \otimes \cdots \otimes x_{2 p+3}\right)\right) \\
& +(-1)^{p+2} m\left(\delta^{p} \varphi\left(x_{1} \otimes \cdots \otimes x_{2 p+1}\right) \otimes x_{2 p+2} \otimes x_{2 p+3}\right) \\
& \quad+\sum_{k=1}^{p+1}(-1)^{k} \delta^{p} \varphi\left(x_{1} \otimes \cdots \otimes m\left(x_{2 k-1} \otimes x_{2 k} \otimes x_{2 k+1}\right) \otimes \cdots \otimes x_{2 p+3}\right) \\
= & m\left(x_{1} \otimes x_{2} \otimes m\left(x_{3} \otimes x_{4} \otimes \varphi\left(x_{5} \otimes \cdots \otimes x_{2 p+3}\right)\right)\right) \\
& +(-1)^{p+1} m\left(x_{1} \otimes x_{2} \otimes m\left(\varphi\left(x_{3} \otimes \cdots \otimes x_{2 p+1}\right) \otimes x_{2 p+2} \otimes x_{2 p+3}\right)\right) \\
& +\sum_{i=1}^{p}(-1)^{i} m\left(x_{1} \otimes x_{2} \otimes \varphi\left(x_{3} \otimes \cdots \otimes m\left(x_{2 i+1} \otimes x_{2 i+2} \otimes x_{2 i+3}\right) \otimes \cdots \otimes x_{2 p+3}\right)\right) \\
& +(-1)^{p+2} m\left(x_{1} \otimes x_{2} \otimes m\left(\varphi\left(x_{3} \otimes \cdots \otimes x_{2 p+1}\right) \otimes x_{2 p+2} \otimes x_{2 p+3}\right)\right) \\
& +(-1)^{2 p+3} m\left(m\left(\varphi\left(x_{1} \otimes \cdots \otimes x_{2 p-1}\right) \otimes x_{2 p} \otimes x_{2 p+1}\right) \otimes x_{2 p+2} \otimes x_{2 p+3}\right) \\
& +(-1)^{p+2} \sum_{i=1}^{p}(-1)^{i} m\left(\varphi\left(x_{1} \otimes \cdots \otimes m\left(x_{2 i-1} \otimes x_{2 i} \otimes x_{2 i+1}\right) \otimes \cdots \otimes x_{2 p+1}\right)\right. \\
& \left.\quad \otimes x_{2 p+2} \otimes x_{2 p+3}\right) \\
& +\sum_{k=1}^{p+1}(-1)^{k} m\left(x_{1} \otimes x_{2} \otimes \varphi\left(x_{3} \otimes \cdots \otimes m\left(x_{2 k-1} \otimes x_{2 k} \otimes x_{2 k+1}\right) \otimes \cdots \otimes x_{2 p+3}\right)\right)
\end{aligned}
$$




$$
\begin{aligned}
& +\sum_{k=1}^{p+1}(-1)^{k}(-1)^{p+1} m\left(\varphi\left(x_{1} \otimes \cdots \otimes m\left(x_{2 k-1} \otimes x_{2 k} \otimes x_{2 k+1}\right) \otimes \cdots \otimes x_{2 p+1}\right)\right. \\
& \left.\otimes x_{2 p+2} \otimes x_{2 p+3}\right) \\
& +\sum_{k=3}^{p+1} \sum_{i=1}^{k-2}(-1)^{k+i} \varphi\left(x_{1} \otimes \cdots \otimes m\left(x_{2 i-1} \otimes x_{2 i} \otimes x_{2 i+1}\right)\right. \\
& \left.\otimes \cdots \otimes m\left(x_{2 k-1} \otimes x_{2 k} \otimes x_{2 k+1}\right) \otimes \cdots \otimes x_{2 p+3}\right) \\
& +\sum_{k=1}^{p}(-1)^{2 k} \varphi\left(x_{1} \otimes \cdots \otimes m\left(m\left(x_{2 k-1} \otimes x_{2 k} \otimes x_{2 k+1}\right) \otimes x_{2 k+2} \otimes x_{2 k+3}\right) \otimes \cdots \otimes x_{2 p+3}\right) \\
& +\sum_{k=1}^{p}(-1)^{2 k+1} \varphi\left(x_{1} \otimes \cdots \otimes m\left(x_{2 k-1} \otimes x_{2 k} \otimes m\left(x_{2 k+1} \otimes x_{2 k+2} \otimes x_{2 k+3}\right)\right) \otimes \cdots \otimes x_{2 p+3}\right) \\
& +\sum_{k=1}^{p-2} \sum_{i=2 k+1}^{p+1}(-1)^{k+i+1} \varphi\left(x_{1} \otimes \cdots \otimes m\left(x_{2 k-1} \otimes x_{2 k} \otimes x_{2 k+1}\right)\right. \\
& \left.\otimes \cdots \otimes m\left(x_{2 i-1} \otimes x_{2 i} \otimes x_{2 i+1}\right) \otimes \cdots \otimes x_{2 p+3}\right)=0
\end{aligned}
$$

\section{Takhtajan's construction}

In this section, we aim to extend to ternary algebras of associative type a process introduced by Takhtajan to construct a complex of ternary algebras starting from a complex of binary algebras (see [56]). Let $(V, m)$ be a ternary algebra of a given type. We associate to it a binary algebra on $W=V \otimes V$ and a map $\Delta$. Assume that $(\mathcal{C}, \delta)$ is a complex for the ternary algebras and $(M, d)$ is a complex for the binary algebras.

We define a map $\Delta$ such that $\Delta_{p}$ associates to any $p$-cochain on $V$ a $p$-cochain on $W$. It is defined by

$$
\begin{array}{ccc}
\Delta_{0}: \mathcal{C}^{0}=\operatorname{Hom}(V, V) & \longrightarrow & M^{0}=\operatorname{Hom}(W, W) \\
f & \longmapsto & \Delta_{0}(f)
\end{array}
$$

such that, for example, $\Delta_{0}(f)\left(x_{1} \otimes x_{2}\right)=f\left(x_{1}\right) \otimes x_{2}+\alpha x_{1} \otimes f\left(x_{2}\right), \forall x_{i} \in V$ with $\alpha \in \mathbb{K}$.

One extends this operation to

$$
\begin{array}{ccc}
\Delta_{p}: \mathcal{C}^{p}=\operatorname{Hom}\left(V^{\otimes 2 p+1}, V\right) & \longrightarrow & M^{p}=\operatorname{Hom}\left(W^{p+1}, W\right) \\
\varphi & \longmapsto & \Delta_{p} \varphi
\end{array}
$$

defined, for example, using the remark that $W^{\otimes p+1} \cong V^{\otimes 2 p+2}$, by

$$
\Delta_{p} \varphi\left(y_{1} \otimes \cdots \otimes y_{2 p+2}\right)=\varphi\left(y_{1} \otimes \cdots \otimes y_{2 p+1}\right) \otimes y_{2 p+2}+\alpha y_{1} \otimes \varphi\left(y_{2} \otimes \cdots \otimes y_{2 p+2}\right)
$$

Let us assume that one has a complex $(M, d)$ :

$$
\cdots \longrightarrow M^{p-1} \stackrel{d^{p-1}}{\longrightarrow} M^{p} \stackrel{d^{p}}{\longrightarrow} M^{p+1} \longrightarrow \cdots
$$

i.e., for all $p, d^{p} \circ d^{p-1}=0$.

Consider for any $p>0$, the linear maps $\delta^{p}: \mathcal{C}^{p} \longrightarrow \mathcal{C}^{p+1}$ satisfying

$$
\Delta_{p+1} \circ \delta^{p}=d^{p} \circ \Delta_{p}, \quad \forall p
$$

The equality is well defined. 
Indeed, one has for $p \geq 1$,

$$
\mathcal{C}^{p} \stackrel{\Delta_{p}}{\longrightarrow} M^{p} \stackrel{d^{p}}{\longrightarrow} M^{p+1} \quad \text { and } \quad \mathcal{C}^{p} \stackrel{\delta^{p}}{\longrightarrow} \mathcal{C}^{p+1} \stackrel{\Delta_{p+1}}{\longrightarrow} M^{p+1}
$$

Lemma 5.1. Let $p>1$. If $d^{p} \circ d^{p-1}=0$, then $\delta^{p} \circ \delta^{p-1}=0$.

Proof. One has $\Delta_{p+1} \circ \delta^{p}=d^{p} \circ \Delta_{p}$, then $\Delta_{p+1} \circ \delta^{p} \circ \delta^{p-1}=d^{p} \circ \Delta_{p} \circ \delta^{p-1}=d^{p} \circ d^{p-1} \circ \Delta_{p-1}=0$, because $d^{p} \circ d^{p-1}=0$.

As a consequence of the previous lemma, one may obtain a complex of ternary algebras starting from a complex of binary algebras and a map $\Delta$. This process was used by Takhtajan to construct a cohomology of ternary Nambu algebras using the Chevalley-Eilenberg cohomology of Lie algebras. The binary multiplication used to that end is defined as follows:

Let $(V,[,]$,$) be a ternary Nambu algebra. Set W=V \otimes V$. The multiplication on $W$ is defined for $x_{1} \otimes x_{2}, y_{1} \otimes y_{2} \in W$ by

$$
\left[x_{1} \otimes x_{2}, y_{1} \otimes y_{2}\right]_{W}=\left[x_{1}, x_{2}, y_{1}\right] \otimes y_{2}+y_{1} \otimes\left[x_{1}, x_{2}, y_{2}\right]
$$

\subsection{Takhtajan's construction and ternary algebras of associative type}

In the sequel, we show that we cannot derive a cohomology of a partially associative ternary algebra from a cohomology of binary algebras of associative type. A construction is possible in the case of totally associative ternary algebras but the cohomology obtained is the cohomology of weak totally associative ternary algebras described above.

A binary algebra is called of associative type if it is given by a vector space $V$ and a multiplication $\mu$ satisfying an identity of the form

$$
\mu(\mu(u \otimes v) \otimes w)+\lambda \mu(u \otimes \mu(v \otimes w))=0
$$

where $\lambda$ is a scalar element different from zero. In particular, we have associative algebras for $\lambda=-1$ and skew-associative algebras for $\lambda=1$. In the last section, we show that the skew-associative algebras cannot carry a usual cohomology adapted to deformation theory.

In the following, we try to adapt the Takhatjan's procedure to ternary algebras of associative type. We set

$$
\mu\left(\left(x_{1} \otimes x_{2}\right) \otimes\left(y_{1} \otimes y_{2}\right)\right)=m\left(x_{1} \otimes x_{2} \otimes y_{1}\right) \otimes y_{2}+\alpha x_{1} \otimes m\left(x_{2} \otimes y_{1} \otimes y_{2}\right)
$$

where $\alpha \in \mathbb{K}$.

In order to check whether $\mu$ is a binary operation of associative type, we compute

$$
\begin{aligned}
A_{1}= & \mu\left(\mu\left(\left(x_{1} \otimes x_{2}\right) \otimes\left(y_{1} \otimes y_{2}\right)\right) \otimes\left(z_{1} \otimes z_{2}\right)\right) \\
= & \mu\left(\left(m\left(x_{1} \otimes x_{2} \otimes y_{1}\right) \otimes y_{2}\right) \otimes\left(z_{1} \otimes z_{2}\right)\right)+\alpha \mu\left(\left(x_{1} \otimes m\left(x_{2} \otimes y_{1} \otimes y_{2}\right)\right) \otimes\left(z_{1} \otimes z_{2}\right)\right) \\
= & m\left(m\left(x_{1} \otimes x_{2} \otimes y_{1}\right) \otimes y_{2} \otimes z_{1}\right) \otimes z_{2}+\alpha m\left(x_{1} \otimes x_{2} \otimes y_{1}\right) \otimes m\left(y_{2} \otimes z_{1} \otimes z_{2}\right) \\
& +\alpha m\left(x_{1} \otimes m\left(x_{2} \otimes y_{1} \otimes y_{2}\right) \otimes z_{1}\right) \otimes z_{2}+\alpha^{2} x_{1} \otimes m\left(m\left(x_{2} \otimes y_{1} \otimes y_{2}\right) \otimes z_{1} \otimes z_{2}\right)
\end{aligned}
$$

and

$$
\begin{aligned}
A_{2}= & \mu\left(\left(x_{1} \otimes x_{2}\right) \otimes \mu\left(\left(y_{1} \otimes y_{2}\right) \otimes\left(z_{1} \otimes z_{2}\right)\right)\right) \\
= & \mu\left(\left(x_{1} \otimes x_{2}\right) \otimes\left(m\left(y_{1} \otimes y_{2} \otimes z_{1}\right) \otimes z_{2}\right)\right)+\alpha \mu\left(\left(x_{1} \otimes x_{2}\right) \otimes\left(y_{1} \otimes m\left(y_{2} \otimes z_{1} \otimes z_{2}\right)\right)\right) \\
= & m\left(x_{1} \otimes x_{2} \otimes m\left(y_{1} \otimes y_{2} \otimes z_{1}\right)\right) \otimes z_{2}+\alpha x_{1} \otimes m\left(x_{2} \otimes m\left(y_{1} \otimes y_{2} \otimes z_{1}\right) \otimes z_{2}\right) \\
& +\alpha m\left(x_{1} \otimes x_{2} \otimes y_{1}\right) \otimes m\left(y_{2} \otimes z_{1} \otimes z_{2}\right)+\alpha^{2} x_{1} \otimes m\left(x_{2} \otimes y_{1} \otimes m\left(y_{2} \otimes z_{1} \otimes z_{2}\right)\right)
\end{aligned}
$$


The multiplication $\mu$ is of associative type if $A_{1}+\lambda A_{2}=0$, that is,

$$
\begin{aligned}
A_{1}+\lambda A_{2}=[ & m\left(m\left(x_{1} \otimes x_{2} \otimes y_{1}\right) \otimes y_{2} \otimes z_{1}\right)+\alpha m\left(x_{1} \otimes m\left(x_{2} \otimes y_{1} \otimes y_{2}\right) \otimes z_{1}\right) \\
& \left.+\lambda m\left(x_{1} \otimes x_{2} \otimes m\left(y_{1} \otimes y_{2} \otimes z_{1}\right)\right)\right] \otimes z_{2} \\
& +\alpha x_{1} \otimes\left[\alpha m\left(m\left(x_{2} \otimes y_{1} \otimes y_{2}\right) \otimes z_{1} \otimes z_{2}\right)+\lambda m\left(x_{2} \otimes m\left(y_{1} \otimes y_{2} \otimes z_{1}\right) \otimes z_{2}\right)\right. \\
& \left.\quad+\alpha \lambda m\left(x_{2} \otimes y_{1} \otimes m\left(y_{2} \otimes z_{1} \otimes z_{2}\right)\right)\right] \\
& +\alpha(1+\lambda) m\left(x_{1} \otimes x_{2} \otimes y_{1}\right) \otimes m\left(y_{2} \otimes z_{1} \otimes z_{2}\right)
\end{aligned}
$$

If $m$ is a ternary operation which defines a partially associative ternary algebra of a given type, then $A_{1}+\lambda A_{2}=0$ if $\alpha(1+\lambda)=0$ and the coefficients $(1, \alpha, \lambda),\left(\alpha^{2}, \lambda \alpha, \lambda \alpha^{2}\right)$ are proportional.

The first condition is satisfied when $\alpha=0$ or $\lambda=-1$. The case $\alpha=0$ is impossible.

If $\lambda=-1$, the coefficients $(1, \alpha,-1)$ and $\left(\alpha^{2},-\alpha,-\alpha^{2}\right)$ should be proportional. This is possible only over $\mathbb{C}$ with $\alpha= \pm i$. The associativity condition needed must be of one of the following forms:

$$
\begin{aligned}
& m\left(m\left(x_{1} \otimes x_{2} \otimes x_{3}\right) \otimes x_{4} \otimes x_{5}\right)+i m\left(x_{1} \otimes m\left(x_{2} \otimes x_{3} \otimes x_{4}\right) \otimes x_{5}\right) \\
& \quad-m\left(x_{1} \otimes x_{2} \otimes m\left(x_{3} \otimes x_{4} \otimes x_{5}\right)\right)=0 \\
& m\left(m\left(x_{1} \otimes x_{2} \otimes x_{3}\right) \otimes x_{4} \otimes x_{5}\right)-i m\left(x_{1} \otimes m\left(x_{2} \otimes x_{3} \otimes x_{4}\right) \otimes x_{5}\right) \\
& \quad-m\left(x_{1} \otimes x_{2} \otimes m\left(x_{3} \otimes x_{4} \otimes x_{5}\right)\right)=0
\end{aligned}
$$

In the both cases, one may construct a cohomology of ternary algebras according to Takhtajan's construction and using the Hochschild complex of associative binary multiplication.

Therefore, we have the following proposition.

Proposition 5.2. It is impossible to construct, using Takhtajan's construction, a cohomology of ternary algebras $(V, m)$ which are partially associative (resp., alternate partially associative) starting from a complex of binary algebra of associative type.

Remark 5.3. If the ternary algebra $m$ is totally associative, then the corresponding binary algebra is of associative type if

$$
1+\alpha+\lambda=0, \quad \alpha^{2}+\lambda \alpha+\lambda \alpha^{2}=0, \quad \alpha(1+\lambda)=0
$$

which implies that $\alpha=0$ and $\lambda=-1$.

Therefore, using Takhtajan procedure, we can construct a cohomology of totally associative ternary algebras $(V, m)$ with a binary multiplication $\mu$ defined on $W=V \otimes V$ by

$$
\mu\left(\left(x_{1} \otimes x_{2}\right) \otimes\left(y_{1} \otimes y_{2}\right)\right)=m\left(x_{1} \otimes x_{2} \otimes y_{1}\right) \otimes y_{2} \quad \forall x_{1} \otimes x_{2}, y_{1} \otimes y_{2} \in W
$$

Let $\varphi: V^{\otimes 2 p-1} \rightarrow V$ be a $p$-cochain of the totally associative ternary algebra $(V, m)$. We set $\Delta \varphi\left(x_{1} \otimes \cdots \otimes x_{2 p}\right)=\varphi\left(x_{1} \otimes \cdots \otimes x_{2 p-1}\right) \otimes x_{2 p}$. It turns out that in this case, we recover the coboundary map of the weak totally associative ternary algebras discussed in Section 4.

Really, using the Hochschild coboundary of the binary associative algebra $(W, \mu)$, we have

$$
\begin{aligned}
d^{p} \Delta & \varphi\left(x_{1} \otimes \cdots \otimes x_{2 p+2}\right) \\
= & \mu\left(x_{1} \otimes x_{2} \otimes \Delta \varphi\left(x_{3} \otimes \cdots \otimes x_{2 p+2}\right)\right) \\
& +\sum_{i=1}^{p} \Delta \varphi\left(x_{1} \otimes \cdots \otimes \mu\left(x_{2 i-1} \otimes x_{2 i} \otimes x_{2 i+1} \otimes x_{2 i+2}\right) \otimes \cdots \otimes x_{2 p+2}\right) \\
& +(-1)^{p} \mu\left(\Delta \varphi\left(x_{1} \otimes \cdots \otimes x_{2 p}\right) \otimes x_{2 p+1} \otimes x_{2 p+2}\right)
\end{aligned}
$$




$$
\begin{aligned}
= & m\left(x_{1} \otimes x_{2} \otimes \varphi\left(x_{3} \otimes \cdots \otimes x_{2 p+1}\right)\right) \otimes x_{2 p+2} \\
& +\sum_{i=1}^{p} \varphi\left(x_{1} \otimes \cdots \otimes m\left(x_{2 i-1} \otimes x_{2 i} \otimes x_{2 i+1}\right) \otimes \cdots \otimes x_{2 p+1}\right) \otimes x_{2 p+2} \\
& +(-1)^{p} m\left(\varphi\left(x_{1} \otimes \cdots \otimes x_{2 p-1}\right) \otimes x_{2 p} \otimes x_{2 p+1}\right) \otimes x_{2 p+2}
\end{aligned}
$$

Then, we set

$$
\begin{aligned}
\delta^{p} \varphi\left(x_{1} \otimes \cdots \otimes x_{2 p+1}\right)= & m\left(x_{1} \otimes x_{2} \otimes \varphi\left(x_{3} \otimes \cdots \otimes x_{2 p+1}\right)\right) \\
& +\sum_{i=1}^{p} \varphi\left(x_{1} \otimes \cdots \otimes m\left(x_{2 i-1} \otimes x_{2 i} \otimes x_{2 i+1}\right) \otimes \cdots \otimes x_{2 p+1}\right) \\
& +(-1)^{p} m\left(\varphi\left(x_{1} \otimes \cdots \otimes x_{2 p-1}\right) \otimes x_{2 p} \otimes x_{2 p+1}\right)
\end{aligned}
$$

and recover the cohomology of weak totally associative ternary algebras defined above.

\section{On deformation cohomology of skew-associative algebras}

In this section, we show that the 1-cohomology and 2-cohomology guided by 1-parameter formal deformations cannot be extended to a usual 3-cohomology. Therefore, the operad of skew-associative binary algebras is not Koszul.

Definition 6.1. A skew-associative binary algebra is given by a $\mathbb{K}$-vector space $V$ and a bilinear multiplication $\mu$ satisfying, for every $x_{1}, x_{2}, x_{3} \in V$,

$$
\mu\left(\mu\left(x_{1} \otimes x_{2}\right) \otimes x_{3}\right)=-\mu\left(x_{1} \otimes \mu\left(x_{2} \otimes x_{3}\right)\right)
$$

The formal deformation theory leads to the following 1-coboundary and 2-coboundary operators for a cohomology of skew-associative binary algebra $\mathcal{A}=(V, \mu)$ adapted to formal deformation theory. The 1-coboundary operator of $\mathcal{A}$ is the map

$$
\delta^{1}: \mathcal{C}^{0}(\mathcal{A}, \mathcal{A}) \longrightarrow \mathcal{C}^{1}(\mathcal{A}, \mathcal{A}), \quad f \longmapsto \delta^{1} f
$$

defined by

$$
\delta^{1} f\left(x_{1} \otimes x_{2}\right)=f\left(\mu\left(x_{1} \otimes x_{2}\right)\right)-\mu\left(f\left(x_{1}\right) \otimes x_{2}\right)-\mu\left(x_{1} \otimes f\left(x_{2}\right)\right)
$$

The 2-coboundary operator of $\mathcal{A}$ the map

$$
\delta^{2}: \mathcal{C}^{1}(\mathcal{A}, \mathcal{A}) \longrightarrow \mathcal{C}^{2}(\mathcal{A}, \mathcal{A}), \quad \varphi \longmapsto \delta^{2} \varphi
$$

defined by

$$
\begin{aligned}
\delta^{2} \varphi\left(x_{1} \otimes x_{2} \otimes x_{3}\right)= & \mu\left(\varphi\left(x_{1} \otimes x_{2}\right) \otimes x_{3}\right)+\mu\left(x_{1} \otimes \varphi\left(x_{2} \otimes x_{3}\right)\right) \\
& +\varphi\left(\mu\left(x_{1} \otimes x_{2}\right) \otimes x_{3}\right)+\varphi\left(x_{1} \otimes \mu\left(x_{2} \otimes x_{3}\right)\right)
\end{aligned}
$$

One may characterize the operator $\delta^{2}$ using the following skew-associator map:

$$
\circ: \mathcal{C}^{r}(\mathcal{A}, \mathcal{A}) \times \mathcal{C}^{s}(\mathcal{A}, \mathcal{A}) \longrightarrow \mathcal{C}^{r+s}(\mathcal{A}, \mathcal{A}), \quad(f, g) \longmapsto f \circ g
$$

defined by

$$
(f \circ g)\left(x_{1} \otimes \cdots \otimes x_{r+s}\right)=\sum_{i=0}^{r-1} f\left(x_{1} \otimes \cdots \otimes g\left(x_{i+1} \otimes \cdots \otimes x_{i+s}\right), \cdots \otimes x_{r+s}\right)
$$

We have $\delta^{2} \varphi=\mu \circ \varphi+\varphi \circ \mu$. Note also that $\delta^{2} \circ \delta^{1}=0$. 
Proposition 6.2. A usual 3-coboundary operator extending the maps $\delta^{1}$ and $\delta^{2}$ to a complex of for skew-associative binary algebras does not exist.

Proof. We set the following general form of a usual 3-coboundary operator:

$$
\begin{aligned}
\delta^{3} f\left(x_{1} \otimes x_{2} \otimes x_{3} \otimes x_{4}\right)= & a_{1} \mu\left(x_{1} \otimes f\left(x_{2} \otimes x_{3} \otimes x_{4}\right)\right)+a_{2} f\left(\mu\left(x_{1} \otimes x_{2}\right) \otimes x_{3} \otimes x_{4}\right) \\
& +a_{3} f\left(x_{1} \otimes \mu\left(x_{2} \otimes x_{3}\right) \otimes x_{4}\right)+a_{4} f\left(x_{1} \otimes x_{2} \otimes \mu\left(x_{3} \otimes x_{4}\right)\right) \\
& +a_{5} \mu\left(f\left(x_{1} \otimes x_{2} \otimes x_{3}\right) \otimes x_{4}\right)
\end{aligned}
$$

We consider a 3-cochain $f$, that is, a map $f: V^{\otimes 3} \rightarrow V$, and a 2-cochain $g$, that is, a map $f: V^{\otimes 2} \rightarrow V$. We compute $\delta^{3}\left(\delta^{2} g\right)\left(x_{1} \otimes x_{2} \otimes x_{3} \otimes x_{4}\right)$ and substitute $\mu\left(y_{1} \otimes \mu\left(y_{2} \otimes y_{3}\right)\right)$ by $-\mu\left(\mu\left(y_{1} \otimes y_{2}\right) \otimes y_{3}\right)$. Then, we obtain

$$
\begin{aligned}
& \left(a_{3}-a_{4}\right) f\left(x_{1} \otimes \mu\left(\mu\left(x_{2} \otimes x_{3}\right) \otimes x_{4}\right)\right)+\left(a_{2}+a_{4}\right) f\left(\mu\left(x_{1} \otimes x_{2}\right) \otimes \mu\left(x_{3} \otimes x_{4}\right)\right) \\
& \quad+\left(a_{2}-a_{3}\right) f\left(\mu\left(\mu\left(x_{1} \otimes x_{2}\right) \otimes x_{3}\right) \otimes x_{4}\right)+\left(a_{1}+a_{4}\right) \mu\left(x_{1} \otimes f\left(x_{2} \otimes \mu\left(x_{3} \otimes x_{4}\right)\right)\right) \\
& \quad+\left(a_{1}+a_{3}\right) \mu\left(x_{1} \otimes f\left(\mu\left(x_{2} \otimes x_{3}\right) \otimes x_{4}\right)\right)+\left(a_{3}+a_{5}\right) \mu\left(f\left(x_{1} \otimes \mu\left(x_{2} \otimes x_{3}\right)\right) \otimes x_{4}\right) \\
& \quad+\left(a_{2}+a_{5}\right) \mu\left(f\left(\mu\left(x_{1} \otimes x_{2}\right) \otimes x_{3}\right) \otimes x_{4}\right)+\left(a_{2}-a_{1}\right) \mu\left(\mu\left(x_{1} \otimes x_{2}\right) \otimes f\left(x_{3} \otimes x_{4}\right)\right) \\
& \quad+\left(a_{5}-a_{1}\right) \mu\left(\mu\left(x_{1} \otimes f\left(x_{2} \otimes x_{3}\right)\right) \otimes x_{4}\right)+\left(a_{5}-a_{4}\right) \mu\left(\mu\left(f\left(x_{1} \otimes x_{2}\right) \otimes x_{3}\right) \otimes x_{4}\right)=0
\end{aligned}
$$

The equation is satisfied for all $x_{1}, x_{2}, x_{3}, x_{4} \in V$ if and only if $a_{i_{\{i=1, \ldots, 5\}}}=0$.

Corollary 6.3. A usual deformation cohomology for skew-associative binary algebras does not exist. Then the operad of skew-associative binary algebras is not Koszul.

\section{References}

[1] V. Abramov, B. Le Roy, and R. Kerner. Hypersymmetry: A Z3-graded generalization of supersymmetry. J. Math. Phys., 38 (1997), 1650-1669.

[2] H. Ataguema and A. Makhlouf. Deformations of ternary algebras. J. Gen. Lie Theory App., 1 (2007), 41-55.

[3] J. Bagger and N. Lambert. Gauge symmetry and supersymmetry of multiple M2-branes. Preprint, arXiv: 0711.0955, 2007.

[4] N. Bazunova, A. Borowiec, and R. Kerner. Universal differential calculus on ternary algebras. Lett. Math. Phys., 67 (2004), 195-206.

[5] A. Borowiec, W. Dudek, and S. Duplij. Basic concepts of ternary Hopf algebras. J. Kharkov Nat. Univ., Ser. Nuclei, Particles and Fields, 529 (2001), 21-29.

[6] R. Carlsson. $n$-ary algebras. Nagoya Math. J., 78 (1980), 45-56.

[7] R. Carlsson. Cohomology of associative triple systems. Proc. Amer. Math. Soc., 60 (1976), 1-7.

[8] J. M. Cassas, J.-L. Loday, and T. Pirashvili. Leibniz n-algebras. Forum Math., 14 (2002), 189-207.

[9] J. A. De Azcarraga, J. M. Izquierdo, and J. C. Perez Bueno. On the higher-order generalizations of Poisson structures. J. Phys. A: Math. Gen., 30 (1997), 607-616.

[10] J. A. De Azcarraga, A. M. Perelomov, and J. C. Perez Bueno. The Schouten-Nijenhuis bracket, cohomology and generalized Poisson structures. J. Phys. A: Math. Gen., 29 (1996), 7993-8009.

[11] G. Dito, M. Flato, D. Sternheimer, and L. Takhtajan. Deformation quantization and Nambu mechanics. Commun. Math. Phys., 183 (1997), 1-22.

[12] G. Dito, M. Flato, and D. Sternheimer. Nambu mechanics, $n$-ary operations and their quantization. In "Deformation Theory and Symplectic Geometry". Math. Phys. Stud., 20, Kluwer Academic Publishers, Dordrecht, Boston, 1997, 43-66. 
[13] V. T. Filippov. n-ary Lie algebras. Sibirskii Math. J., 24 (1985), 126-140.

[14] P. Gautheron. Some remarks concerning Nambu mechanics. Lett. Math. Phys., 37 (1996), 103-116.

[15] P. Gautheron. Simple facts concerning Nambu algebras. Comm. Math. Phys., 195 (1998), $417-434$.

[16] M. Gerstenhaber. On the deformations of rings and algebras I. Ann. of Math., 79 (1964), 59-103.

[17] M. Gerstenhaber. On the deformations of rings and algebras II. Ann. of Math., 84 (1966), 1-19.

[18] M. Gerstenhaber. On the deformations of rings and algebras III. Ann. of Math., 88 (1968), 1-34.

[19] V. Ginzburg and M. Kapranov. Koszul duality for operads. Duke Math. J., 76 (1994), 203-272.

[20] A. V. Gnedbaye. Les algèbres K-aires et leurs opérades. C. R. Acad. Sci. Paris. Série I, 321 (1995), 147-152.

[21] A. V. Gnedbaye. Opérades, algèbres de Leibniz et triples de Jordan. Mémoire de synthèse pour l'habilitation à diriger des recherches, IRMA Strasbourg Preprint, 2003.

[22] N. Goze and E. Remm. On $n$-ary algebras given by Gerstenhaber's products. Preprint, arXiv: 0803.0553v2 [math.RA], 2008.

[23] M. Goze and M. Rausch de Traubenberg. Hopf algebras for ternary algebras and groups. Preprint, arXiv: 0809.4212v1 [math-ph], 2008.

[24] J. Grabowski and G. Marmo. Remarks on Nambu-Poisson and Nambu-Jacobi brackets. J. Phys. A: Mah. Gen., 32 (1999), 4239-4247.

[25] Ph. Hanlon and M. Wachs. On Lie k-algebras. Adv. Math., 113 (1995), 206-236.

[26] B. Harris. Cohomology of Lie triple systems and Lie algebras with involution. Trans. Amer. Math. Soc., 98 (1961), 148-162.

[27] M. R. Hestenes. On ternary algebras. Scripta Math., 29 (1973), 253-272.

[28] E. Hoffbeck. A Poincaré-Birkhoff-Witt criterion for Koszul operads. Preprint, arXiv: 0709.2286v3 [math.AT], 2007.

[29] N. Jacobson. Lie and Jordan triple systems. Amer. J. Math., 71 (1949), 149-170.

[30] M. Kapranov, M. Gelfand, and A. Zelevinskii. Discriminants, Resultants and Multidimensional Determinants. Birkhäuser, Berlin, 1994.

[31] R. Kerner. Ternary algebraic structures and their applications in physics. In "Proc. BTLP 23rd International Colloquium on Group Theoretical Methods in Physics". Preprint, arXiv: math$\mathrm{ph} / 0011023,2000$.

[32] R. Kerner. Z3-graded algebras and non-commutative gauge theories. In "Spinors, Twistors, Clifford Algebras and Quantum Deformations". Z. Oziewicz, B. Jancewicz, and A. Borowiec, Eds. Kluwer Academic Publishers, Dordrecht, Boston, 1993, 349-357.

[33] R. Kerner. Z3-grading and ternary algebraic structures. In "New Symmetries and Differential Geometry". V. Dobrev, M. D. Doebner, and S. Ushveridze, Eds. World Scientific, Singapore, 1994, 375-394.

[34] R. Kerner. The cubic chessboard. Class. Quantum Grav., 14 (1997), A203-A225.

[35] R. Kerner. $Z_{3}$-graded ternary algebras, new gauge theories and quarks. In "Topics in Quantum Field Theory". T. Tchrakian, Ed. World Scientific, Singapore, 1995, 113-126.

[36] R. Kerner and L. Vainerman. On special classes of $n$-algebras. J. Math. Phys., 37 (1996), 2553-2565.

[37] R. Kerner. Generalized cohomologies and differentials of higher order. Proc. DGMTP Con. M.-L. Ge and W. Zhang, Eds. World Scientific, Singapore, 2006.

[38] F. Kubo and Y. Taniguchi. A controlling cohomology of deformation theory of Lie triple systems. J. Algebra, 278 (2004), 242-250.

[39] A. G. Kurosh. Multioperator rings and algebras. Russian Math. Surv., 24 (1969), 1-13.

[40] W. G. Lister. Ternary rings. Trans. Amer. Math. Soc., 154 (1971), 37-55.

[41] J.-L. Loday. Cyclic Homology. Springer-Verlag, Berlin, 1992.

[42] O. Loos. Assoziative tripelsysteme. Manuscripta Math., 7 (1972), 103-112.

[43] O. Loos. Symmetric spaces I: General Theory. Benjamin, New York, 1969. 
[44] A. Makhlouf. A comparison of deformations and geometric study of varieties of associative algebras. Int. J. Math. and Math. Sci., 2007 (2007), 1-24.

[45] M. Markl, S. Shnider, and J. Stasheff. Operads in Algebra, Topology and Physics. Mathematical Surveys and Monographs, 96, American Mathematical Society, Providence, RI, 2002.

[46] P. W. Michor and I. Vaisman. A note on $n$-ary Poisson brackets. Rend. Circ. Mat. Palermo (2) Suppl., 63 (2000), 165-172.

[47] P. W. Michor and A. M. Vinogradov. $n$-ary Lie and assciative algebras. Rend. Sem. Mat. Univ. Pol. Torino., 54 (1996), 373-392.

[48] Y. Nambu. Generalized Hamiltonian mechanics. Phys. Rev., D7 (1973), 2405-2412.

[49] S. Okubo. Triple products and Yang-Baxter equation (I): Octonionic and quaternionic triple systems. J. Math. Phys., 34 (1993), 3273-3291.

[50] M. Rausch de Traubenberg and M. J. Slupinski. Finite-dimensional Lie algebras of order F. J. Math. Phys., 43 (2002), 5145-5160.

[51] M. Rausch de Traubenberg. Ternary algebras and groups. J. Phys.: Conf. Ser., 128 (2008), 012060 .

[52] M. Rausch de Traubenberg. Some results on cubic and higher order extensions of the Poincaré algebra. Preprint, arXiv: 0811.1465v1 [hep-th], 2008.

[53] E. Remm. On the NonKoszulity of $(2 p+1)$-ary partially associative operads. Preprint, arXiv: 0812.2687v1 [math.AT], 2008.

[54] N. P. Sokolov. Introduction to the Theory of Multidimensional Matrices. Naukova Dumaka, Kiev, 1972.

[55] L. Takhtajan. On foundation of the generalized Nambu mechanics. Comm. Math. Phys., 160 (1994), 295-315.

[56] L. Takhtajan. Higher order analog of Chevally-Eilenberg complex and deformation theory of $n$-algebras. St. Petersburg Math. J., 6 (1995), 429-438.

[57] K. Yamaguti. On the cohomologie space of Lie triple systems. Kumamoto J. Sci. Ser. A, 5 (1960), $44-52$.

[58] Ch. A. Weibel. An Introduction to Homological Algebra. Cambridge Studies in Advanced Mathematics 38, Cambridge University Press, 1994.

Received January 15, 2009 